\title{
Desempenho de Diferentes Grupos Genéticos de Bovinos de Corte em Confinamento
}

\author{
Kepler Euclides Filho1, Geraldo Ramos de Figueiredo², Valéria Pacheco Batista Euclides ${ }^{2}$, \\ Luiz Otávio Campos da Silva ${ }^{2}$, Vanessa Rocco ${ }^{3}$, Rodrigo Amorim Barbosa ${ }^{4}$, Claudia Elias Junqueira ${ }^{5}$
}

\begin{abstract}
RESUMO - Foram utilizadas informações provenientes de 188 animais, pertencentes a dez grupos genéticos, avaliados em confinamento. Para as análises dos dados, os animais foram agrupados em três subconjuntos considerando-se idade, sexo e dieta recebida. Os subconjuntos analisados foram: 1) animais inteiros de sobreano recebendo a dieta "a": 39 Nelore (N), 12 Brangus (BR), 8 1/2 Simental1/2 Nelore (SN), 8 1/2 Caracu-1/2 Nelore (CCN), 21 1/2 Valdostana-1/2 Nelore (VAN); 2) animais inteiros desmamados recebendo a dieta "b": 12 N, 12 1/2 Canchim-1/4 Angus-1/4 Nelore (CAN), 16 1/2 Canchim-1/4 Simental-1/4 Nelore (CSN), 12 1/2 Braford-1/2 Brangus (BDBR), 12 1/2 Braford-1/4 Angus-1/4 Nelore (BDAN), 7 1/2 Brahman-1/4 Angus-1/4 Nelore (BHAN); 3) fêmeas desmamadas recebendo a dieta "b" em duas formulações, uma em que o concentrado representou $30 \%$ da MS e o outro, em que o concentrado correspondeu a 50\% da MS total. Neste caso, a análise incluiu 29 fêmeas, 15 CAN e 14 CSN. Para os animais dos subconjuntos 1 e 2 , o concentrado foi fornecido para atender $50 \%$ da MS total da dieta. Os animais do subconjunto 1 apresentaram desempenhos semelhantes, com ganho de peso diário médio igual a 1,60 kg/dia e com conversão alimentar igual a 6,36 kg de MS ingerida/kg de ganho de peso. Para o ganho de peso diário médio e para a conversão alimentar, somente foram observadas diferenças entre os animais do subconjunto 2 . $\mathrm{O}$ maior ganho de peso diário médio foi observado para os animais CSN (1,69 kg/dia) e as melhores conversões alimentares foram verificadas para os animais CSN e BHAN (4,76 kg de MS ingerida $/ \mathrm{kg}$ de ganho de peso e 4,67 kg de MS ingerida $/ \mathrm{kg}$ de ganho de peso, respectivamente). Para o subconjunto 3 não houve diferenças entre os grupos genéticos, mas a formulação da dieta apresentou efeitos significativos importantes, especialmente, para conversão alimentar. Os animais da formulação com menor teor de concentrado apresentaram melhor conversão alimentar (5,58 kg de MS ingerida/kg de ganho de peso versus 6,66 kg de MS ingerida/ $\mathrm{kg}$ de ganho de peso).
\end{abstract}

Palavras-chave: conversão alimentar, cruzamento, eficiência bionutricional, ganho de peso

\section{Performance Evaluation of Different Beef Cattle Genetic Groups under Feedlot}

\begin{abstract}
It was utilized data from 188 animals from ten genetic groups. For the statistical analysis, the animals were grouped into three subgroups according to age, sex and diet. Thus, the subgroups analyzed were: 1) intacted yearling males receiving the "a" ration: 39 Nellore (N), 12 Brangus (BR), 8 1/2 Simmental - 1/2 Nellore (SN), 8 1/2 Caracu - 1/2 Nellore (CCN), 21 1/2 Valdostana - 1/2 Nellore (VAN); 2) intacted weaned males receiving the "b" diet: 12 N, 12 1/2 Canchim - 1/2 1/4 Angus - 1/4 Nellore (CAN), 16 1/2 Canchim - 1/4 Simmental - 1/4 Nellore (CSN), 12 Braford - 1/2 Brangus (BRBD), 12 1/2 Braford - 1/4 Angus - 1/4 Nellore (BDAN), 7 Brahman - 1/4 Angus - 1/4 Nellore (BHAN); 3) weaned females receiving the " $b$ " diet under two formulations, one in which the concentrate represented $30 \%$ of total dry matter (DM) and the other in which the participation of the concentrate was $50 \%$ of total DM. For this subgroup the analysis included 29 females, 15 CAN and 14 CSN. Animals in subgroups 1 and 2, were fed a diet contained $50 \%$ of concentrate in the DM. Animals in subgroup 1 had similar performances. The average daily gain was $1.60 \mathrm{~kg} /$ day and average feed conversion was $6.41 \mathrm{~kg}$ of DM intake/kg of weight gain. The statistical analysis revealed that for average daily gain as well as for feed conversion, it were observed differences only among animals on subgroup 2 . The greater average daily gain was recorded on CSN animals ( $1.69 \mathrm{~kg}$ /day) and the best feed conversion on CSN and BHAN animals (4.76 kg of DM intake/kg of weight gain and $4.67 \mathrm{~kg}$ of DM intake/ $\mathrm{kg}$ of weight gain). The analyses of subgroup 3 allowed the conclusion that in spite of not having any difference between genetic groups, formulation showed an important effect, mainly on feed conversion. The animals receiving the diet formulation in which the concentrate represented $30 \%$ of the total DM showed better feed conversion $(5.58 \mathrm{~kg}$ of DM intake/kg of weight gain versus $6.66 \mathrm{~kg}$ of DM intake/ $\mathrm{kg}$ of weight gain).
\end{abstract}

Key Words: bio-nutritional efficiency, crossbreeding, feed conversion, weight gain

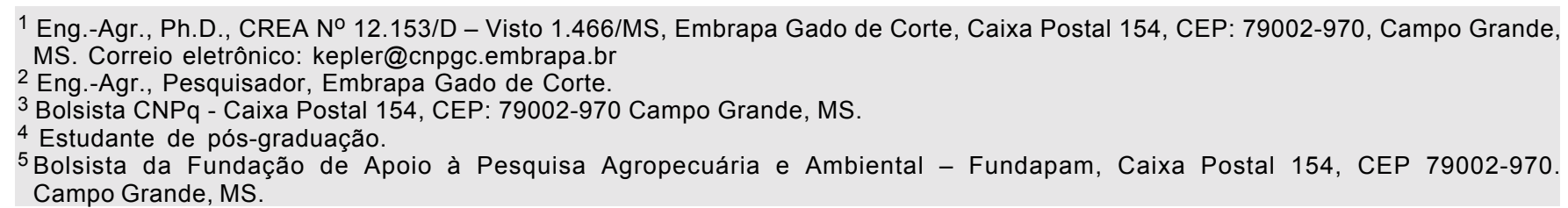




\section{Introdução}

O aumento da competitividade com outras carnes bem como com outros mercados e a possibilidade de o Brasil se consolidar no mercado mundial de carne bovina, têm requerido da atividade de pecuária de corte a oferta de produto de qualidade de maneira contínua durante o ano. Esta demanda juntamente com a necessidade de se aumentar a eficiência do setor têm sido os grandes motores do processo de restruturação em curso na cadeia produtiva da carne bovina. Como parte deste novo cenário, surge a necessidade não só de se avaliar alternativas tecnológicas inovadoras que sejam compatíveis com as novas demandas, mas que se reavaliem algumas tecnologias compatíveis com essa ótica moderna. Nesse sentido, verifica-se que o processo de intensificação pelo qual vem passando a pecuária de corte brasileira tem resultado, entre outros, no aumento da prática de confinamento como alternativa de terminação de animais. Neste contexto, cresce a participação dos denominados novilhos superprecoces, ou seja, animais abatidos com idade aproximada de 12 meses. Estes animais juntamente com os denominados precoces, que são aqueles abatidos com, aproximadamente, dois anos de idade, têm sido os grandes responsáveis pela oferta de produtos de qualidade durante boa parte do ano. Todavia, existe a necessidade de se buscar soluções tecnológicas que sejam capazes de complementar a oferta anual do produto ao mesmo tempo que garantam a rentabilidade dos sistemas de produção.

Para isto, podem ser utilizadas diversas estratégias que vão desde o uso adequado do potencial genético dos animais até a utilização de estratégias alimentares, passando pela combinação de machos e fêmeas para produção de carne. Com respeito ao potencial genético, a utilização de animais com diferentes taxas de maturação pode se constituir em uma estratégia importante, conforme Euclides Filho (2000). Neste mesmo sentido, a combinação de machos e fêmeas, por possuírem taxas de maturação diferentes, pode ser uma alternativa viável para produção de carne o ano todo. Outra alternativa que merece avaliação é o controle de ingestão de alimentos que tem se mostrado capaz de produzir carne bovina de forma bioeconomicamente eficiente. Este controle pode ser realizado tanto pela restrição alimentar e subseqüente capitalização no ganho compensatório, como pela diminuição da densidade energética da dieta de parte dos animais. Quanto à primeira maneira, ou seja, uso do ganho compensatório, resultados de vários trabalhos têm possibilitado concluir-se que a restrição na ingestão de alimentos apesar de produzir crescimento anormal nos animais, resulta em maiores taxas de crescimento durante o período de realimentação quando comparados com animais que foram alimentados normalmente (O'Donovan, 1984, Owens et al., 1993). No entanto, esta estratégia deve ser analisada cuidadosamente, uma vez que, segundo Euclides et al. (1998), este ganho compensatório é, na maioria das vezes, parcial. Com relação à segunda alternativa, restrição energética, esta é, segundo Mersmann (1987), uma das maneiras de se aumentar a eficiência do crescimento animal é diminuir sua ingestão de energia, alimentando-o com níveis inferiores àqueles observados sob alimentação ad libitum.

Neste contexto, o confinamento, por ser uma tecnologia que requer altos investimentos, especialmente em alimentação, demanda o uso de animais eficientes na transformação do alimento consumido em carne. Esta busca por eficiência tem resultado, não só em aumento da utilização dos cruzamentos, mas também no incremento do número de grupos genéticos utilizados e avaliados. Assim, torna-se importante que se conduzam avaliações envolvendo diferentes grupos genéticos, em condições semelhantes. Nesta ótica, diversos trabalhos vêm sendo conduzidos e os resultados encontrados na literatura sugerem diferenças em conversão alimentar ou em eficiência bionutricional entre grupos genéticos de bovinos de corte (Gonçalves et al., 1991, Perotto et al., 1998, Euclides Filho et al., 2001). Tais diferenças podem, em última instância, representar diferenças econômicas importantes entre sistemas de produção.

Este trabalho foi estruturado com o intuito de subsidiar a oferta de carne de qualidade durante o ano todo de forma competitiva. Para tanto, seus objetivos foram avaliar o desempenho de diversos grupos genéticos durante a fase de terminação em confinamento, o uso de fêmeas como estratégia para os sistemas de produção e a redução da densidade energética da ração de parte dos animais como ferramenta para expansão do período de oferta de carne durante $o$ ano. O trabalho foi conduzido focando, basicamente, três aspectos relacionados com a produção e a avaliação de três tipos de animais, quais sejam, machos e fêmeas denominados superprecoces e machos precoces. As fêmeas foram avaliadas sob duas dietas que se diferiam em densidade energética

R. Bras. Zootec., v.32, n.5, p.1114-1122, 2003 
com o intuito de retardar o abate e com isto, ampliar o período de oferta de carne de qualidade. Esta ampliação de período é importante, especialmente, considerando-se que produção de carne bovina no Brasil, apesar de estar em franca transformação, ainda apresenta períodos definidos de safra e entressafra que tem como conseqüência a inexistência de carne de qualidade em todos os meses do ano.

\section{Material e Métodos}

Foram utilizadas informações de 188 animais de dez grupos genéticos distribuídos em dezessete piquetes, de acordo com a idade, sexo e dieta recebida. De acordo com os objetivos, estes animais foram agrupados em três subconjuntos, o primeiro, representando os animais precoces, era constituído por machos de sobreano, aproximadamente, 18 meses de idade, inteiros, confinados na segunda seca de suas vidas, o segundo formou o grupo dos chamados superprecoces, constituídos por machos inteiros confinados após a desmama que ocorreu quando os animais tinham, aproximadamente, seis meses de idade, e o terceiro, formado por fêmeas, também confinadas após a desmama, recebendo porém, dietas em duas formulações que se diferiam quanto à sua densidade energética. Um grupo de fêmeas recebeu uma formulação em que o concentrado representava $50 \%$ da MS total, enquanto para o outro o concentrado constituía $30 \%$ da MS total. Para os dois grupos de machos, a proporção de concentrado na MS total da dieta foi a mesma e igual a $50 \%$. No entanto, as dietas possuíam formulações diferentes de acordo com o potencial de crescimento dos animais, uma vez que o grupo de animais jovens se encontrava na parte da curva onde o crescimento é mais intenso. Os animais de sobreano receberam uma dieta composta por silagem de milho e um concentrado com a seguinte composição: $71 \%$ de milho moído, $25 \%$ de farelo de soja, $2,22 \%$ de uréia, $1,5 \%$ de calcário calcítico, $0,22 \%$ de sulfato de amônio e $0,06 \%$ de Rumensin ${ }^{\circledR}$. Esta dieta foi denominada dieta "a". Os machos desmamados, por sua vez, foram alimentados com uma dieta formada por silagem de milho e por um concentrado constituído por $42,4 \%$ de milho moído, $56 \%$ de farelo de soja, $1,58 \%$ de calcário calcítico e $0,02 \%$ de Rumensin ${ }^{\circledR}$. Esta dieta foi denominada dieta " $b$ ". As fêmeas formaram dois grupos que receberam a dieta "b" em duas formulações. Para um grupo de fêmeas foi fornecido o concentrado de modo a corresponder a $30 \%$ da MS total, enquanto para o outro este concentrado participou com $50 \%$.

Assim, os subconjuntos utilizados nas análises estatísticas foram: 1) machos inteiros de sobreano recebendo a dieta "a": 39 Nelore $(\mathrm{N}), 12$ Brangus (BR), 8 1/2 Simental-1/2 Nelore (SN), 8 1/2 Caracu$1 / 2$ Nelore (CCN), 21 1/2 Valdostana-1/2 Nelore (VAN); 2) machos inteiros desmamados recebendo a dieta "b": 12 Nelore (N), 12 1/2 Canchim-1/4 Angus-1/4 Nelore (CAN), 16 1/2 Canchim-1/4 Simental-1/4 Nelore (CSN), 12 1/2 Braford-1/2 Brangus (BDBR), 12 1/2 Braford-1/4 Angus-1/4 Nelore (BDAN), 7 1/2 Brahman-1/4 Angus-1/4 Nelore (BHAN); e 3 ) fêmeas desmamadas recebendo a dieta "b" em duas formulações. Neste caso, a análise incluiu 29 fêmeas 15 CAN (oito recebendo a dieta com $30 \%$ de concentrado na base da MS e sete recebendo a dieta com $50 \%$ de concentrado) e 14 CSN (sete em cada uma das formulações, $30 \%$ e $50 \%$ de concentrado) .

Todos animais foram criados em pastagens de Brachiaria decumbens, com as mães, até a desmama. Aqueles confinados para produção do chamado "superprecoce" foram, após a desmama, levados para baias coletivas, separados por grupo genético, onde permaneceram por um período de adaptação de, aproximadamente, quatorze dias. Para evitar os efeitos de competição por alimentos e, especialmente, os de dominância, os animais foram acompanhados diariamente, para que se pudesse interferir retirando os animais que apresentassem tais comportamentos. No entanto, nenhum animal foi retirado por este motivo, talvez pelo fato de que em cada baia coletiva, foram colocados animais que haviam sido criados juntos. Além disto, havia ampla disponibilidade de cocho para cada animal, aproximadamente, $1 \mathrm{~m} /$ animal. Todos os animais foram mantidos em confinamento até o abate, que era realizado à medida que eles atingiam o ponto de avaliação, prédefinido como sendo de, aproximadamente, 5 milímetros de gordura de cobertura na carcaça. Este ponto de terminação era aferido por meio visual realizado por duas pessoas treinadas. Todas as avaliações foram realizadas pelas mesmas pessoas. A quantidade de ração diária era ajustada a cada três dias de maneira a garantir uma sobra, aproximada, de $10 \%$ do fornecido. A cada 28 dias todos animais eram pesados após jejum de sólidos e líquidos de 16 horas. 
Para análise dos dados de ganho de peso e conversão alimentar foram utilizados os três subconjuntos: 1) os machos de sobreano; 2) os machos desmamados; 3 ) as fêmeas que receberam as duas formulações (30 e 50\% de concentrado na MS total). Foi utilizado um modelo matemático geral que continha os efeitos fixos de grupo genético, nível de concentrado na dieta e sexo. Idade e peso no início da avaliação foram incluídos no modelo como covariáveis. Esse modelo geral foi ajustado para cada conjunto de dados, e interações simples foram utilizadas nos subconjuntos onde eram apropriadas.

\section{Resultados e Discussão}

Os resumos das análises de variância encontram-se nas Tabelas 1, 2 e 3. Nas Tabelas 4 e 5, são apresentadas as médias de ganho de peso diário médio e de conversão alimentar para os animais dos subconjuntos 1 e 2 . Para análise das fêmeas, por estas terem sido avaliadas em duas dietas, foi utilizado o modelo completo.

A análise estatística dos diversos subconjuntos permitiu verificar que tanto para o ganho de peso diário médio, quanto para a conversão alimentar, somente foram observadas diferenças importantes entre os animais do subconjunto $2(\mathrm{P}>0,0001)$.

Os animais do subconjunto 1 (Tabela 4) apresentaram desempenhos semelhantes com ganho de peso diário médio igual a, aproximadamente, 1,60 quilogra$\mathrm{ma} /$ dia e conversão alimentar média de, aproximadamente, $6,36 \mathrm{~kg}$ de MS ingerida/kg de ganho de peso.
Todavia, ao se analisar o consumo de matéria seca verificaram-se diferenças significativas entre os diversos grupos genéticos (Tabela 4), o que mais uma vez, pode estar sugerindo a possibilidade de a conversão alimentar não ser uma boa característica para comparação entre animais de potenciais diferentes, como afirmaram Euclides Filho et al. (2000, 2001). Segundo estes autores, correlações entre consumo de matéria seca e ganho de peso podem não ser lineares entre os diversos grupos genéticos e a conversão alimentar não seria capaz de considerar tais diferenças. A eficiência bionutricional, definida por Euclides Filho et al. (2001), por outro lado, por considerar consumo de MS diário e ganho de peso em uma análise bivariada, poderia ser mais indicada para tais comparações. Os animais VAN (8,55 kg de MS/ dia) apresentaram menor consumo e os animais $\mathrm{SN}$, tendência de maior consumo (10,71 kg de MS/dia). A análise da eficiência bionutricional revelou diferenças entre os grupos genéticos, sendo que os animais VAN apresentaram desempenho superior aos demais mestiços $(\mathrm{P}<0,050)$, não sendo, contudo, diferentes dos animais $\mathrm{N}(\mathrm{P}<0,0834)$. Não houve diferenças $(\mathrm{P}<0,7777)$ entre os demais animais $(\mathrm{N}, \mathrm{BR}, \mathrm{SN}$ e $\mathrm{CCN})$. Os valores de eficiência bionutricional para os diversos grupos genéticos do subconjunto 1 foram: 3,$17 ; 3,24 ; 3,39 ; 3,19$ e 2,79 para N, BR, SN, CCN e VAN, respectivamente.

Estes valores, relativamente altos de consumo, possivelmente, estejam associados ao ponto de avaliação de $5 \mathrm{~mm}$ de gordura de cobertura que resultaram em pesos de abate entre 480 e $490 \mathrm{~kg}$. Ítavo et

Tabela 1 - Resumo da análise de variância para a conversão alimentar, o ganho de peso diário médio, o consumo diário médio e a eficiência bionutricional dos animais do subconjunto 1

Table 1 - Summary of the analyses of variance for feed conversion, average daily gain, average daily intake and bio-nutritional efficiency for animals in subgroup 1

\begin{tabular}{|c|c|c|c|c|c|}
\hline \multirow[b]{2}{*}{$\begin{array}{l}\text { Fonte de variação } \\
\text { Source of variation }\end{array}$} & \multirow[b]{2}{*}{$\begin{array}{l}\mathrm{GL} \\
D F\end{array}$} & \multicolumn{4}{|c|}{$\begin{array}{l}\text { Quadrados médios } \\
\text { Mean squares }\end{array}$} \\
\hline & & $\begin{array}{c}\text { Conversão } \\
\text { alimentar } \\
\text { Feed } \\
\text { conversion }\end{array}$ & $\begin{array}{l}\text { Ganho diário } \\
\text { médio } \\
\text { Average } \\
\text { dailygain }\end{array}$ & $\begin{array}{c}\text { Consumo } \\
\text { diário médio } \\
\text { Average } \\
\text { daily intake }\end{array}$ & $\begin{array}{c}\text { Eficiência } \\
\text { bionutricional } \\
\text { Bio-nutritional } \\
\text { efficiency }\end{array}$ \\
\hline Grupo genético & 4 & 1,18887 & 0,06966 & $4,9870^{* *}$ & $0,38073^{* *}$ \\
\hline $\begin{array}{l}\text { Genetic group } \\
\text { Idade inicial } \\
\text { Initial age }\end{array}$ & 1 & 1,12124 & 0,00740 & 0,1858 & 0,00024 \\
\hline $\begin{array}{l}\text { Peso inicial } \\
\text { Initial weight }\end{array}$ & 1 & $8,73470^{*}$ & 0,20317 & $0,8758^{*}$ & 0,08667 \\
\hline $\begin{array}{l}\text { Erro } \\
\text { Error }\end{array}$ & 81 & 1,56558 & 0,09331 & 0,2051 & 0,10836 \\
\hline
\end{tabular}

R. Bras. Zootec., v.32, n.5, p.1114-1122, 2003 
Tabela 2 - Resumo da análise de variância para a conversão alimentar, o ganho de peso diário médio, o consumo diário médio e a eficiência bionutricional dos animais do subconjunto 2

Table 2 - Summary of the analyses of variance for feed conversion, average daily gain, average daily intake and bio-nutritional efficiency for animals in subgroup 2

\begin{tabular}{|c|c|c|c|c|c|}
\hline \multirow[b]{2}{*}{$\begin{array}{l}\text { Fonte de variação } \\
\text { Source of variation }\end{array}$} & \multirow[b]{2}{*}{$\begin{array}{l}\text { GL } \\
D F\end{array}$} & \multicolumn{4}{|c|}{$\begin{array}{l}\text { Quadrados médios } \\
\text { Mean squares }\end{array}$} \\
\hline & & $\begin{array}{l}\text { Conversão } \\
\text { alimentar } \\
\text { Feed } \\
\text { convertion }\end{array}$ & $\begin{array}{c}\text { Ganho diário } \\
\text { médio } \\
\text { Average } \\
\text { daily gain }\end{array}$ & $\begin{array}{c}\text { Consumo } \\
\text { diário médio } \\
\text { Average } \\
\text { daily intake }\end{array}$ & $\begin{array}{c}\text { Eficiência } \\
\text { bionutricional } \\
\text { Bio-nutritional } \\
\text { efficiency }\end{array}$ \\
\hline Grupo genético & 5 & $2,71791^{* *}$ & $0,14617^{* *}$ & $4,54710^{* *}$ & $1,05633^{* *}$ \\
\hline $\begin{array}{l}\text { Genetic group } \\
\text { Idade inicial } \\
\text { Initial age }\end{array}$ & 1 & 0,77753 & 0,05744 & 0,04663 & 0,09260 \\
\hline $\begin{array}{l}\text { Peso inicial } \\
\text { Initial weight }\end{array}$ & 1 & $3,65214^{* *}$ & 0,09164 & $0,67815^{*}$ & 0,00140 \\
\hline $\begin{array}{l}\text { Erro } \\
\text { Error }\end{array}$ & 63 & 0,42321 & 0,02305 & 0,14664 & 0,04404 \\
\hline
\end{tabular}

Tabela 3 - Resumo da análise de variância para a conversão alimentar, o ganho de peso diário médio, o consumo diário médio e a eficiência bionutricional dos animais do subconjunto 3

Table 3 - Summary of the analyses of variance for feed conversion, average daily gain, average daily intake and bio-nutritional efficiency for animals in subgroup 3

\begin{tabular}{|c|c|c|c|c|c|}
\hline \multirow[b]{2}{*}{$\begin{array}{l}\text { Fonte de variação } \\
\text { Source of variation }\end{array}$} & \multirow[b]{2}{*}{$\begin{array}{l}\text { GL } \\
D F\end{array}$} & \multicolumn{4}{|c|}{$\begin{array}{c}\text { Quadrados médios } \\
\text { Mean squares }\end{array}$} \\
\hline & & $\begin{array}{c}\text { Conversão } \\
\text { alimentar } \\
\text { Feed } \\
\text { conversion }\end{array}$ & $\begin{array}{c}\text { Ganho diário } \\
\text { médio } \\
\text { Average } \\
\text { dailygain }\end{array}$ & $\begin{array}{c}\text { Consumo } \\
\text { diário médio } \\
\text { Average } \\
\text { daily intake }\end{array}$ & $\begin{array}{c}\text { Eficiência } \\
\text { bionutricional } \\
\text { Bio-nutritional } \\
\text { efficiency }\end{array}$ \\
\hline $\begin{array}{l}\text { Grupo genético }(\mathrm{GG}) \\
\text { Genetic group }(G G)\end{array}$ & 1 & 0,53290 & 0,00619 & 0,03764 & $0,07404^{* *}$ \\
\hline $\begin{array}{l}\operatorname{Dieta}(\mathrm{D}) \\
\operatorname{Diet}(D)\end{array}$ & 1 & $2,72656^{*}$ & 0,01009 & $5,30610^{* *}$ & $4,69206^{* *}$ \\
\hline $\begin{array}{l}\mathrm{GG} \times \mathrm{D} \\
G G \times D\end{array}$ & 1 & 0,44161 & 0,00179 & 0,00914 & $0,56031^{* *}$ \\
\hline $\begin{array}{l}\text { Idade inicial } \\
\text { Initial age }\end{array}$ & 1 & 0,08829 & 0,00279 & 0,00887 & 0,00039 \\
\hline $\begin{array}{l}\text { Peso inicial } \\
\text { Initial weight }\end{array}$ & 1 & 0,26940 & 0,00443 & 0,00264 & 0,00061 \\
\hline $\begin{array}{l}\text { Erro } \\
\text { Error }\end{array}$ & 23 & 0,53426 & 0,01626 & 0,02839 & 0,00226 \\
\hline
\end{tabular}

al. (2002), avaliando o consumo e a digestibilidade de animais Nelore na fase de terminação, verificaram que o consumo máximo foi de 7,97 e $6,93 \mathrm{~kg} / \mathrm{dia}$ em dietas com níveis de concentrado iguais a 40 e $60 \%$, respectivamente, no entanto, neste caso, os animais eram abatidos com $450 \mathrm{~kg}$ de peso vivo. Com relação à conversão alimentar, os valores do presente trabalho estão melhores do que os obtidos por Backes et al. (2001), trabalhando com animais Santa Gertrudis. Estes autores observaram consumo médio igual a 7,44 kg de MS/kg de ganho. Esta variação nos resultados de consumo e de conversão alimentar é relativamente comum na literatura, uma vez que são vários os fatores que influenciam estas variáveis. Capelle et al. (2001), em uma revisão de trabalhos envolvendo avaliação de consumo e ganho de peso, relataram valores médios de, aproximadamente, 9,0 $\mathrm{kg} / \mathrm{dia}$ para animais Nelore e de, aproximadamente, $10,40 \mathrm{~kg} / \mathrm{dia}$ para animais "meio-sangue" Europeu-Nelore, com ganhos de peso de 0,85 e 1,00 kg/dia, respectivamente.

Com relação ao subconjunto 2 (Tabela 5), a 
Tabela 4 - Médias de quadrados mínimos para ganho de peso médio diário e para conversão alimentar, de acordo com o grupo genético, para os animais do subconjunto 1

Table 4 - Least squares means for average daily gain and feed conversion, according to genetic group, for the animals from subgroup 1

\begin{tabular}{|c|c|c|c|}
\hline $\begin{array}{l}\text { Grupo genético } \\
\text { Genetic group }\end{array}$ & $\begin{array}{c}\text { Ganho de } \\
\text { peso, kg } \\
\text { Weight gain, } k g\end{array}$ & $\begin{array}{c}\text { Conversão alimentar, } \\
\mathrm{kg} \mathrm{MS} / \mathrm{kg} \text { ganho } \\
\text { Feed conversion, } \mathrm{kg} \mathrm{DM} / \mathrm{kg} \\
\text { weight gain }\end{array}$ & $\begin{array}{c}\text { Consumo diário } \\
\text { médio de } \mathrm{MS}, \mathrm{kg} / \mathrm{dia} \\
\text { Average daily } \\
\text { intake, } \mathrm{kg} / \text { day }\end{array}$ \\
\hline & $\mathrm{P}>0,5632$ & $\mathrm{P}>0,5547$ & $\mathrm{P}>0,0001$ \\
\hline $\begin{array}{l}\text { Nelore }(\mathrm{N}) \\
\text { Nellore }(N)\end{array}$ & 1,59 & 6,48 & $10,01^{\mathrm{a}}$ \\
\hline Brangus (BR) & 1,62 & 6,70 & $10,21^{\text {ad }}$ \\
\hline $\begin{array}{l}\text { Brangus }(B R) \\
1 / 2 \text { Simental-1/2 Nelore (SN) } \\
1 / 2 \text { Simmental }-1 / 2 \text { Nellore }(S N)\end{array}$ & 1,69 & 6,77 & $10,71^{\mathrm{d}}$ \\
\hline $\begin{array}{l}\text { 1/2 Caracu-1/2 Nelore (CCN) } \\
1 / 2 \text { Caracu }-1 / 2 \text { Nellore }(C C N)\end{array}$ & 1,62 & 6,13 & $9,87^{\mathrm{ab}}$ \\
\hline $\begin{array}{l}\text { 1/2 Valdostana- } 1 / 2 \text { Nelore (VAN) } \\
1 / 2 \text { Valdostana }-1 / 2 \text { Nellore }(V A N)\end{array}$ & 1,44 & 5,73 & $8,55^{\mathrm{c}}$ \\
\hline
\end{tabular}

Tabela 5 - Médias de quadrados mínimos para ganho de peso médio diário e para conversão alimentar, de acordo com o grupo genético, para os animais do subconjunto 2

Table 5 - Least squares means for average daily gain and feed conversion, according to genetic group, for the animals from subgroup 2

\begin{tabular}{|c|c|c|c|}
\hline $\begin{array}{l}\text { Grupo genético } \\
\text { Genetic group }\end{array}$ & $\begin{array}{c}\text { Ganho de } \\
\text { peso, kg } \\
\text { Weight gain, kg }\end{array}$ & $\begin{array}{c}\text { Conversão alimentar, } \\
\mathrm{kg} \mathrm{MS} / \mathrm{kg} \text { ganho } \\
\text { Feed conversion, } \mathrm{kg} \mathrm{DM} / \mathrm{kg} \\
\text { weight gain }\end{array}$ & $\begin{array}{c}\text { Consumo diário } \\
\text { médio de } \mathrm{MS}, \mathrm{kg} / \mathrm{dia} \\
\text { Average daily } \\
\text { intake, } \mathrm{kg} / \text { day }\end{array}$ \\
\hline & $\mathrm{P}>0,0001$ & $\mathrm{P}>0,0001$ & $\mathrm{P}>0,0001$ \\
\hline $\begin{array}{l}\text { Nelore }(\mathrm{N}) \\
\text { Nellore }(N)\end{array}$ & $1,30^{\mathrm{a}}$ & $5,92^{\mathrm{a}}$ & $7,61^{\mathrm{a}}$ \\
\hline $\begin{array}{l}\text { 1/2 Braford }-1 / 2 \text { Brangus (BDBR) } \\
1 / 2 \text { Braford }-1 / 2 \text { Brangus (BDBR) }\end{array}$ & $1,45^{\mathrm{a}}$ & $5,73^{\mathrm{a}}$ & $8,15^{b}$ \\
\hline $\begin{array}{l}\text { 1/2 Braford-1/4 Angus-1/4 Nelore (BDAN) } \\
1 / 2 \text { Braford - } 1 / 4 \text { Angus - } 1 / 4 \text { Nellore (BDAN) }\end{array}$ & $1,35^{\mathrm{a}}$ & $5,99^{\mathrm{a}}$ & $7,85^{\mathrm{c}}$ \\
\hline $\begin{array}{l}\text { 1/2 Brahman-1/4 Angus-1/4 Nelore (BHAN) } \\
1 / 2 \text { Brahman - } 1 / 4 \text { Angus - } 1 / 4 \text { Nellore (BHAN) }\end{array}$ & $1,33^{\mathrm{a}}$ & $4,67^{\mathrm{b}}$ & $6,08^{c}$ \\
\hline $\begin{array}{l}\text { 1/2 Canchim-1/4 Angus-1/4 Nelore (CAN) } \\
1 / 2 \text { Canchim - } 1 / 4 \text { Angus - } 1 / 4 \text { Nellore (CAN) }\end{array}$ & $1,48^{\mathrm{a}}$ & $5,46^{\mathrm{a}}$ & $8,08^{a b}$ \\
\hline $\begin{array}{l}\text { 1/2 Canchim-1/4 Simental-1/4 Nelore (CSN) } \\
\text { 1/2 Canchim - 1/4 Simmental - 1/4 Nellore (CSN) }\end{array}$ & $1,69^{\mathrm{b}}$ & $4,76^{\mathrm{b}}$ & $8,25^{\text {bd }}$ \\
\hline
\end{tabular}

superioridade para conversão alimentar observada para os animais CSN não era esperada uma vez que esses animais possuíam, em sua composição racial, maior participação de raças de grande tamanho adulto e, por isto, apresentam maior exigência de mantença, conforme pode ser verificada na ampla revisão apresentada no National Research Council (1996). Esse resultado também está em discordância com aqueles obtidos por Euclides Filho et al. (2001) que verificaram melhor desempenho nutricional para animais 1/2 Canchim-1/4 Angus-1/4 Nelore do que para 1/2 Canchim-1/4 Simental-1/2 Nelore ou 1/2 PardoSuíço-1/2 Nelore.

O maior ganho de peso diário médio foi observado para os animais CSN (1,69 kg/dia) e as melhores conversões alimentares foram registradas para os animais CSN e BHAN (4,76 kg de MS ingerida $/ \mathrm{kg}$ de ganho de peso e 4,67 kg de MS ingerida $/ \mathrm{kg}$ de ganho de peso, respectivamente). A melhor conversão alimentar dos animais BHAN pode, possivelmente, ser explicada pelo menor consumo de MS diário verificado para estes animais $(6,08 \mathrm{~kg}$ de $\mathrm{MS} / \mathrm{dia})$. O desem- 
penho do grupo CSN, por outro lado, provavelmente, esteja relacionado com o maior ganho de peso verificado para este grupo, uma vez que o seu consumo de MS foi bem mais elevado, ou seja, $8,25 \mathrm{~kg}$ de MS/ dia (Tabela 5). Os consumos verificados pelos animais deste subconjunto foram superiores àquele observado por Costa et al. (2002) que variaram de 6,90 $\mathrm{kg} / \mathrm{dia}$ a $6,94 \mathrm{~kg} /$ dia quando o peso de abate foi de 340 $\mathrm{kg}$ para $430 \mathrm{~kg}$. Neste caso, muito possivelmente, as diferenças sejam também provenientes do ponto de acabamento utilizado, uma vez que segundo estes autores, os animais apresentaram 3,91 $\mathrm{mm}$ e 4,63 $\mathrm{mm}$, de gordura de cobertura, respectivamente, para os abatidos com $340 \mathrm{~kg} \mathrm{e} 430 \mathrm{~kg}$ de peso vivo. Veloso et al. (2002), por outro lado, trabalhando com animais $1 / 2$ Simental - $1 / 2$ Nelore, em uma dieta com $50 \%$ de concentrado com base na matéria seca, registraram consumo de MS igual a 7,52 kg/dia.

Todavia, é importante ressaltar a possibilidade de estes grupos genéticos apresentarem diferenças nas correlações entre consumo de MS e ganho de peso. Por isto, foi conduzida uma análise avaliando-se a eficiência bionutricional. O uso deste índice, de fato, possibilitou a detecção de diferenças entre os grupos genéticos. A melhor eficiência bionutricional foi observada para os animais BHAN $(3,57)$, seguida daquela registrada para os animais $N(4,13)$ que apresentaram desempenho superior àqueles observados para os animais BDBR, CAN e CSN (4,47; 4,48 e 4,74 respectivamente). Os animais CAN e BDBR foram superiores aos CSN.

Maior eficiência na utilização de alimentos por animais mais precoces foi também observada por Gregory et al. (1994). O desempenho dos animais Nelore neste subconjunto também foi contrastante com diversos outros resultados encontrados na literatura. Barbosa (1998) encontrou, como resultado de uma ampla revisão, diferenças entre animais puros, "meio-sangue" e cruzados de três raças. Tais diferenças favorecem os animais oriundos de cruzamentos de três raças e estão respaldadas no fato de nesses animais, se observarem efeitos das heteroses direta e materna.

Tendências de melhores conversões alimentares para animais oriundos de cruzamentos envolvendo a raça Angus foram verificadas nos subconjuntos 2 e 3 . No subconjunto 2, a melhor conversão alimentar foi verificada nos animais cuja composição racial envolvia a participação das raças Angus e Brahman e Nelore (Tabela 5).

Para o subconjunto 3 não foram observadas diferenças, apesar de haver ligeira tendência a favor dos animais com $25 \%$ de participação de raça precoce na sua composição racial, CAN. Estes animais apresentaram conversão alimentar igual a 5,97 quilogramas de MS ingerida/quilograma de ganho de peso, enquanto os CSN necessitaram consumir 6,27 quilogramas de MS para cada quilograma de ganho de peso (Tabela 6). Tendência de melhor conversão alimentar para animais oriundos de cruzamentos entre Bos taurus e Bos indicus foi também constatada pela revisão de Barbosa (1998). Este autor verificou ain$\mathrm{da}$, que as conversões alimentares eram melhores quando os cruzamentos envolviam raças britânicas.

Para o subconjunto 3 , não houve efeito significativo $(\mathrm{P}>0,05)$ da interação grupo genético $\mathrm{x}$ nível de concentrado sobre o ganho de peso diário médio ou sobre a conversão alimentar e por isto, os resultados foram discutidos considerando-se grupo genético e formulação das dietas. Para estes dois efeitos também não se observaram diferenças para ganho de peso diário médio, consumo de MS ou para conversão alimentar entre os grupos genéticos $(\mathrm{P}<0,5433$, $\mathrm{P}<0,2609$ e $\mathrm{P}<0,3283$, respectivamente). A média para o ganho de peso foi de $1,14 \mathrm{~kg} / \mathrm{dia}$, enquanto

Tabela 6 - Médias de quadrados mínimos para ganho de peso diário médio, para conversão alimentar e para consumo diário médio de MS dos animais do subconjunto 3 , de acordo com o grupo genético

Table 6 - Least squares means for average daily gain and feed conversion for animals from subgroup 3, according to genetic group

\begin{tabular}{lccc}
\hline $\begin{array}{l}\text { Grupo genético } \\
\text { Genetic group }\end{array}$ & $\begin{array}{c}\text { Ganho de } \\
\text { peso, kg } \\
\text { Weight gain, } \mathrm{kg}\end{array}$ & $\begin{array}{c}\text { Conversão alimentar, } \\
\mathrm{kg} \mathrm{MS} / \mathrm{kg} \text { ganho } \\
\text { Feed conversion, } \mathrm{kg} \mathrm{DM} / \mathrm{kg} \\
\text { weight gain }\end{array}$ & $\begin{array}{c}\text { Consumo diário } \\
\text { médio de MS, kg/dia } \\
\text { Average daily } \\
\text { intake, } \mathrm{kg} / \text { day }\end{array}$ \\
\hline & $\mathrm{P}>0,5433$ & $\mathrm{P}>3283$ & $\mathrm{P}>0,2609$ \\
\hline $\begin{array}{l}1 / 2 \text { Canchim-1/4 Angus-1/4 Nelore }(\mathrm{CAN}) \\
1 / 2 \text { Canchim }-1 / 4 \text { Angus }-1 / 4 \text { Nellore }(\mathrm{CAN})\end{array}$ & 1,16 & 5,97 & 6,93 \\
$1 / 2$ Canchim $-1 / 4$ Simental-1/4 Nelore $(\mathrm{CSN})$ & $1 / 4$ Simmental $-1 / 4$ Nellore $(\mathrm{CSN})$ & 6,27 & 7,01 \\
\hline
\end{tabular}

R. Bras. Zootec., v.32, n.5, p.1114-1122, 2003 
aquela para o consumo de MS foi de $6,96 \mathrm{~kg}$ de $\mathrm{MS} /$ dia e a para conversão alimentar foi de 6,12 $\mathrm{kg}$ de MS ingerida/kg de ganho de peso (Tabela 6). Novamente, a análise da eficiência bionutricional mostrou-se capaz de revelar diferenças não identificadas pela conversão alimentar. Neste caso, a interação entre dieta e grupo genético foi significativa $(\mathrm{P}<0,0001)$, no entanto, observou-se que esta significância foi resultante da não-linearidade das diferenças entre o desempenho dos dois grupos genéticos nas duas dietas e, portanto, pode-se concluir que houve diferença entre os dois grupos de fêmeas, com as CAN apresentando melhor desempenho do que as CSN $(\mathrm{P}<0,0001)$.

Na Tabela 7 encontram-se as médias de quadrados mínimos para ganho de peso, consumo de MS e conversão alimentar de acordo com as duas formulações da dieta "b" utilizadas para as fêmeas (30 e $50 \%$ de concentrado com base na MS). Apesar de ter havido diferenças significativas $(\mathrm{P}<0,0337)$ para conversão alimentar, não houve diferença entre as duas dietas para ganho de peso $(\mathrm{P}<0,4388)$. A média de ganho de peso para os animais nas duas dietas foi de, aproximadamente, $1,14 \mathrm{~kg} / \mathrm{dia}$. É possível que a inexistência de diferenças para ganho de peso nas dietas com diferentes níveis energéticos tenha sido resultante de mudanças na composição de ganho provocadas pela dieta com $50 \%$ de concentrado na MS. Possivelmente, em especial para novilhas, a quantidade de energia fornecida tenha sido acima daquela requerida para a deposição de proteína, o que aumentou a taxa de deposição de gordura. Como conseqüência, há redução da taxa de ganho de peso. De fato, Owens et al. (1995) concluíram, após extensa revisão, que a relação proteína:gordura na carcaça pode ser aumentada reduzindo-se a ingestão de energia durante o período de crescimento, ou mesmo, durante a fase de engorda. Por outro lado, no entanto, houve diferença $(\mathrm{P}<0,0001)$ entre os consumos de MS. Os animais que receberam a dieta em que o concentrado participou com $50 \%$ da MS total consumiram mais do que aqueles que receberam a dieta com $30 \%$ de concentrado (Tabela 7). Possivelmente, esta menor ingestão tenha ocorrido como conseqüência da ação do mecanismo de controle físico de consumo, conforme Mertens (1994).

As fêmeas que receberam a dieta com $30 \%$ de concentrado na MS apresentaram melhor conversão alimentar (Tabela 7). Este resultado é reflexo do menor consumo de MS verificado na dieta menos energética (Tabela 7). Todavia, as fêmeas recebendo a dieta com menor teor de concentrado permaneceram, aproximadamente, 20 dias a mais no confinamento para atingirem o mesmo ponto de avaliação, ou seja, aproximadamente, $5 \mathrm{~mm}$ de gordura de cobertura. Este resultado sugere que a estratégia de se utilizar diferentes teores de concentrado na dieta pode ser uma alternativa para se ampliar o período de oferta de carne de qualidade. Há necessidade, entretanto, que se procedam avaliações econômicas para dar suporte ao uso desta estratégia alimentar. Neste caso, o resultado da eficiência bionutricional foi semelhante àquele observado pela conversão alimentar, ou seja, os animais CAN apresentaram melhor desempenho do que os CSN $(5,96$ versus 6,07 , respectivamente).

Tabela 7 - Médias de quadrados mínimos para ganho de peso diário médio, para conversão alimentar e para consumo diário médio de MS dos animais do subconjunto 3 , de acordo com a dieta

Table 7 - Least squares means for average daily gain, feed conversion and daily dry matter intake for the animals from subgroup 3 , according to the diet

\begin{tabular}{|c|c|c|c|}
\hline $\begin{array}{l}\text { Dieta } \\
\text { Diet }\end{array}$ & $\begin{array}{c}\text { Ganho de } \\
\text { peso, kg } \\
\text { Weight gain, kg }\end{array}$ & $\begin{array}{c}\text { Conversão alimentar, } \\
\mathrm{kg} \mathrm{MS} / \mathrm{kg} \text { ganho } \\
\text { Feed conversion, } \mathrm{kg} \mathrm{DM} / \mathrm{kg} \\
\text { weight gain }\end{array}$ & $\begin{array}{c}\text { Consumo diário } \\
\text { médio de } \mathrm{MS}, \mathrm{kg} / \mathrm{dia} \\
\text { Average daily } \\
\text { intake, } \mathrm{kg} / \text { day }\end{array}$ \\
\hline & $\mathrm{P}>4388$ & $\mathrm{P}>0,0337$ & $\mathrm{P}>0,0001$ \\
\hline $\begin{array}{l}\text { Dieta com } 50 \% \text { de concentrado na MS } \\
\text { Diet with } 50 \% \text { concentrate on DM }\end{array}$ & 1,18 & $6,66^{\mathrm{a}}$ & $7,71^{\mathrm{a}}$ \\
\hline $\begin{array}{l}\text { Dieta com } 30 \% \text { de concentrado na MS } \\
\text { Diet with } 30 \% \text { concentrate on DM }\end{array}$ & 1,11 & $5,58^{\mathrm{b}}$ & $6,22^{\mathrm{b}}$ \\
\hline
\end{tabular}




\section{Conclusões}

O uso de ração com menor teor de concentrado na MS total para animais confinados após a desmama pode ser uma boa alternativa para ampliar o período de oferta de carne de qualidade.

A alternativa de se combinar o controle de ingestão de alimentos com sexo e grupo genético deve ser mais amplamente avaliada.

Sistemas de cruzamentos terminais envolvendo três ou mais raças podem se constituir em importantes componentes de sistemas de produção de bovinos de corte modernos.

A expansão dos cruzamentos, do uso de suplementação alimentar em pasto e do confinamento, requerem avaliações mais detalhadas dos grupos genéticos mais importantes, com relação à eficiência bioeconômica na utilização de alimentos.

\section{Literatura Citada}

BACKES, A.A.; SANCHEZ, L.M.B.; GONÇALVES, M.B.F. Desempenho de novilhos Santa Gertrudis confinados submetidos a dietas com diferentes fontes protéicas e silagem de milho, com ou sem inoculante. Revista Brasileira de Zootecnia, v.30, n.6S, p.2121-2125, 2001.

BARBOSA, P.F. Cruzamentos industriais e a produção de novilhos precoces. In: SIMPÓSIO SOBRE PRODUÇÃO INTENSIVA DE GADO DE CORTE, 1998, Campinas. Anais... Campinas: Colégio Brasileiro de Nutrição Animal, 1998. p.100-114.

CAPELLE, E.R.; VALADARES FILHO, S.C.; SILVA, J.F.C. et al. Estimativas do consumo e do ganho de peso de bovinos em condições brasileiras. Revista Brasileira de Zootecnia, v.30, n.6, p.1857-1865, 2001.

COSTA, E.C.; RESTLE, J.; PASCOAL, L.L. et al. Desempenho de novilhos Red Angus superprecoces, confinados e abatidos com diferentes pesos. Revista Brasileira de Zootecnia, v.31, n.1, p.129-138. 2002.

EUCLIDES FILHO, K. Produção de bovinos de corte e o trinômio genótipo-ambiente-mercado. Campo Grande, Embrapa Gado de Corte, 2000. 61p. (Embrapa Gado de Corte. Documentos, 85)

EUCLIDES FILHO, K.; FIGUEIREDO, G.R.; EUCLIDES, V.P.B. et al. Eficiência bionutricional de animais mestiços de raças européias e Nelore. Revista Brasileira de Zootecnia, v.31, n.1, p.77-82, 2001.

EUCLIDES FILHO, K.; FIGUEIREDO, G.R.; EUCLIDES, V.P.B. et al. Eficiência bionutricional de animais Nelore e seus mestiços com Caracu, Angus e Simental. In: REUNIÃO ANUAL DA SOCIEDADE BRASILEIRA DE ZOOTECNIA, 37., 2000, Viçosa, MG. Anais... Viçosa: Sociedade Brasileira de Zootecnia, 2000. p.219.
EUCLIDES, V.P.B.; EUCLIDES, K.; ARRUDA, Z.J. et al. Desempenho de novilhos em pastagens de Brachiaria decumbens submetidos a diferentes regimes alimentares. Revista da Sociedade Brasileira de Zootecnia, v. 27, n. 2, p. 246-254, 1998.

GONÇALVES, L.C.; SILVA, J.F.C.; ESTEVÃO, M.M. et al. Consumo e digestibilidade da matéria seca e da energia e zebuínos, seus mestiços e bubalinos. Revista da Sociedade Brasileira de Zootecnia, v.20, n.4, p.384-395, 1991.

GREGORY, K.E.; CUNDIFF, L.V.; KOCH, R.M. Breed effects, dietary energy density effects, and retained heterosis on different measures of gain efficiency in beef cattle. Journal of Animal Science, v.72, n.5, p.1138-154, 1994.

ÍTAVO, C.V.L.; VALADARES FILHO, S.C.; SILVA, F.F. et al. Níveis de concentrado e proteína bruta na dieta de bovinos Nelore nas fases de recria e terminação: consumo e digestibilidade. Revista Brasileira de Zootecnia, v. 31, n.2, p.1033-104, 2002.

MERSMANN, H.J. Nutritional and endocrinological influences on the composition of animal growth. Program Food Nutrition Science, v.11, n.2, p.175-201, 1987.

MERTENS, D.R. Regulation of forage intake. In: FAHEY JR., G.C. (Ed.) Forage quality evaluation and utilization. Madison: American Society of Agronomy/Crop Science Society of America/Soil Science Society of America, 1994. p.450-493.

NATIONAL RESEARCH COUNCIL - NRC. Nutrient requirements of beef cattle. 7.ed. Washington, D.C. National Academy Press, 1996. 242p.

O'DONOVAN, P.B. Compensatory gain in cattle and sheep. Nutrition Abstract Review, v.4, n.8, p.389-410, 1984.

OWENS, F.N.; DUBESKI, P.; HANSON, C.F. Factors that alter the growth and development of ruminants. Journal of Animal Science, v.71, n.12, p.3138-3150, 1993.

OWENS, F.N.; GILL, D.R.; SECRIST, D.S. et al. Review of some aspects of growth and development of feedlot cattle. Journal of Animal Science, v.73, n.10, p.3152-3172, 1995.

PEROTTO, D.; CUBAS, A.C.; MOLETTA. J.L. et al. Pesos ao nascimento e à desmama e ganho de peso médio diário do nascimento à desmama de bovinos Charolês, Caracu e cruzamentos recíprocos. Revista Brasileira de Zootecnia, v.27, n.4, p.730-737, 1998.

VELOSO, C.M.; VALADARES FILHO, S.C.; GESUALDI JR., A. et al. Composição corporal e exigências energéticas e protéicas de bovinos F1 Limousin x Nelore não-castrados, alimentados com rações contendo diferentes níveis de concentrado. Revista Brasileira de Zootecnia, v.31, n.3, p.1273-1285, 2002.

Recebido em: 31/05/02 Aceito em: 06/01/03 